\title{
ESSKA partners and the IOC join forces to improve children $A C L$ treatment
}

\author{
Romain Seil $^{1,2} \cdot$ Daniel Theisen $^{2} \cdot$ Håvard Moksnes $^{3}$ (1) $\cdot$ Lars Engebretsen ${ }^{3,4}$
}

Received: 6 February 2018 / Accepted: 28 February 2018 / Published online: 5 March 2018

(c) European Society of Sports Traumatology, Knee Surgery, Arthroscopy (ESSKA) 2018

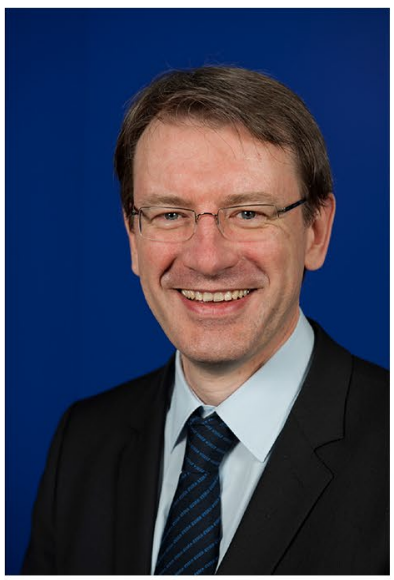

Romain Seil

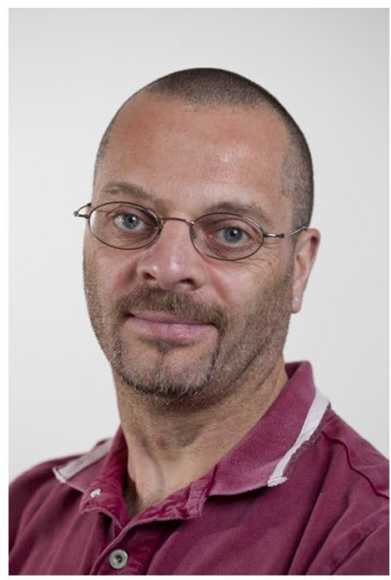

Daniel Theisen

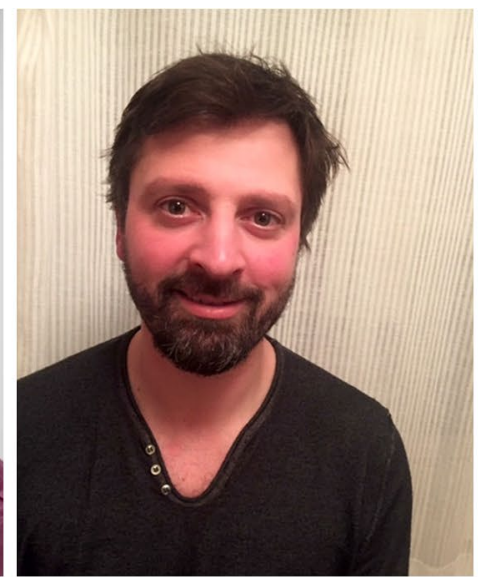

Håvard Moksnes

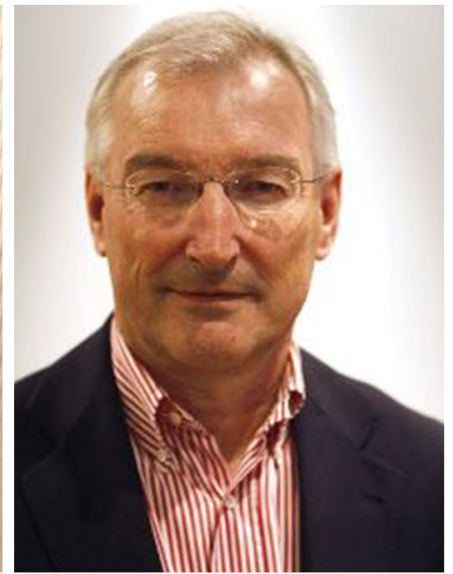

Lars Engebretsen

\section{PAMI project history: what, how and when}

In this issue of KSSTA, we present the consensus paper recently organized by the International Olympic Committee, AOSSM, ISAKOS, SLARD, and ESSKA regarding the challenging issue of anterior cruciate injuries in children [1]. This paper will help our members in their approach to these patients. The consensus paper, put together by 20 experts from all over the world after a Delphi process developed the

Håvard Moksnes

havard.moksnes@olympiatoppen.no

1 Department of Orthopaedic Surgery, Centre Hospitalier Luxembourg, Clinique d'Eich. 78, rue d'Eich, 1460 Luxembourg, Luxembourg

2 Sports Medicine Research Laboratory, Luxembourg Institute of Health, 78 rue d'Eich, 1460 Luxembourg, Luxembourg

3 Oslo Sports Trauma Research Center, Norwegian School of Sport Sciences, PB 4014, Ullevål Stadion, 0806 Oslo, Norway

4 Orthopedic Department, Oslo University Hospital, Kirkeveien 166, 0401 Oslo, Norway
12 questions felt to be the most important in this field, highlights current solutions in these patients, although it is obvious that much knowledge is currently still lacking. There is at least $25 \%$ reinjuries or injuries involving the noninjured knee. Through the "PAMI" initiative, ESSKA and IOC aim to improve the situation.

\section{First steps of the PAMI project}

The ESSKA Paediatric Anterior Cruciate Ligament Monitoring Initiative (PAMI) was started in 2014 by the ESSKA Board in response to the limited evidence-base available on how to treat these injuries. A grant through IOC's Olympic Solidarity to the Luxembourg National Olympic Committee (NOC) and support from Smith \& Nephew made the project possible. An initial step analysed the treatment algorithms and surgical techniques used among ESSKA members [2]. Based on close to 500 respondents ( $22 \%$ of invited) to an e-survey, it was found that paediatric ACL injuries seemed to be more frequent than previously considered, and that treatment practice was highly diverse. The next logical stage 
was to develop an outcome register that would allow for a systematic and standardised data collection within a prospective multicentre approach. Thus, the main purpose of the PAMI project is to create a novel pan-European system to collect and analyse data from orthopaedic surgeons who are treating children and adolescents with anterior cruciate ligament (ACL) injury. The ultimate, specific goals of the project are (1) to describe current treatment options following a paediatric ACL injury, (2) to analyse the associated short-, medium- and long-term clinical outcome, thereby (3) extending the evidence base on optimal treatment choices and, eventually, (4) to propose international treatment guidelines.

\section{Gearing up}

Following the ESSKA Congress in Barcelona in 2016, a formal partnership was established with the Sports Medicine Research Laboratory of the Luxembourg Institute of Health (LIH), a group of scientists with experience in both ACLinjury research and development of custom-made register solutions. A particular challenge in designing the methodology of the PAMI project was to guarantee that all ethical and legal requirements would be met within this international, multicentre research project. At this time, the protocol has been officially cleared by the Luxembourg Research Ethics Committee (CNER), and the notification file addressed to the Luxembourgish data protection agency (CNPD).

The principles applied within the PAMI project comply with the new Regulation 2016/679 (EU) of the European Parliament and of the Council, in place since 24 May 2016, on the protection of natural persons with regard to the processing of personal data and on the free movement of such data. Thus, only pseudonymised patient information will be uploaded into the PAMI database, to ensure maximal data protection. As a consequence, considerable responsibility will lie with partner institutions joining the project. Each has to seek separate ethical clearance, in accordance with their respective national legislation. Acting as local data administrators, nominated on-site coordinators will manage patient information and consent retrieval, store a confidential correspondence table between patient ID and the primary key, handle long-term patient follow-up, and guarantee data access on patient request. Compliance with the security measures put in place to access the PAMI database is compulsory. The PAMI platform has undergone extensive testing over the past months, and the first patients will be recruited during the first trimester of 2018.

\section{Project management}

ESSKA acts as the overall project coordinator and manages the PAMI project via a dedicated steering committee. The latter is composed of at least four members, including one representative from ESSKA (ESSKA being the Data Controller) and one representative from LIH (Data Processor). Currently, the committee is composed of ESSKA president Romain Seil, ESSKA past president Lars Engebretsen, Daniel Theisen representing LIH, Håvard Moksnes, the PAMI coordinator, as well as Kristian Samuelsson as independent observer. All members of the PAMI steering committee are recognised for their clinical or/and scientific expertise in the thematic. Partner institutions/hospitals from different European countries providing treatment to the patient group of interest can engage in this project by filing a formal application to the PAMI coordinator (specific information will be made public soon). They will provide data on current surgical and non-surgical treatments, follow-up treatments, and clinical outcome using the dedicated platform.

The PAMI project will provide important insights into the current outcomes of ACL-injured knees in children, and will eventually allow to discriminate those patients needing operative treatment from those who benefit most from a non-operative treatment. Furthermore, large-scale objective outcome data will provide the knowledgebase necessary for a first-time-ever proposal of international treatment guidelines.

PAMI will be demonstrated and discussed during a symposium on Friday May 11th during the 2018 ESSKA congress in Glasgow. Interested partners are welcome to join the dedicated session from 3 to 4 p.m.

\section{Reference}

1. International Olympic Committee 2018 consensus statement on the management of paediatric anterior cruciate ligament injuries. https://doi.org/10.1007/s00167-018-4865-y

2. Moksnes H, Engebretsen L, Seil R (2016) The ESSKA paediatric anterior cruciate ligament monitoring initiative. Knee Surg Sports Traumatol Arthrosc 24(3):680-687. https://doi.org/10.1007/s0016 7-015-3746-x 